\title{
Criminologie
}

\section{La relance des bacheliers en criminologie de 1970 à 1983}

\section{Michèle Dionne}

Volume 19, numéro 1, 1986

Politiques et pratiques pénales. 25 ans de réflexion et d'action

URI : https://id.erudit.org/iderudit/017236ar

DOI : https://doi.org/10.7202/017236ar

Aller au sommaire du numéro

Éditeur(s)

Les Presses de l'Université de Montréal

ISSN

0316-0041 (imprimé)

1492-1367 (numérique)

Découvrir la revue

Citer cet article

Dionne, M. (1986). La relance des bacheliers en criminologie de 1970 à 1983.

Criminologie, 19(1), 261-279. https://doi.org/10.7202/017236ar

\section{Résumé de l'article}

What is the situation on the labour market today for graduates of the School of Criminology who obtained their bachelor degrees from 1970 on? Based on the answers to a questionnaire covering the 14 years concerned, the professional careers of 546 of the 1001 graduates are traced and described. Of these, $48 \%$ are men and $52 \%$ women, $48 \%$ being between the ages of 25 and 30 . The survey shows that two out of three graduates have jobs directly related to their training, that their principal employer is the civil service and that there is less room in the workplace for women than for men.
Ce document est protégé par la loi sur le droit d'auteur. L'utilisation des services d'Érudit (y compris la reproduction) est assujettie à sa politique d'utilisation que vous pouvez consulter en ligne.

https://apropos.erudit.org/fr/usagers/politique-dutilisation/ 


\section{LA RELANCE DES BACHELIERS EN CRIMINOLOGIE DE 1970 À 1983* \\ Michèle Dionne**}

What is the situation on the labour market today for graduates of the School of Criminology who obtained their bachelor degrees from 1970 on? Based on the answers to a questionnaire covering the 14 years concerned, the professional careers of 546 of the 1001 graduates are traced and described. Of these, $48 \%$ are men and $52 \%$ women, $48 \%$ being between the ages of 25 and 30. The survey shows that two out of three graduates have jobs directly related to their training, that their principal employer is the civil service and that there is less room in the workplace for women than for men.

\section{INTRODUCTION}

L'École de criminologie célèbre en 1985 , ses 25 ans d'existence. Créé en 1960 pour offrir une formation de niveau supérieur, le Département de criminologie offre dès 1967, un programme d'étude de premier cycle. Dès 1970 , les premiers bacheliers ${ }^{1}$ en criminologie se présentaient sur le marché du travail.

Après 14 promotions de baccalauréat, il nous est apparu intéressant de prendre un certain recul afin de situer ces bacheliers quant à leur pratique professionnelle. Cette recherche se propose essentiellement de faire le point sur leur situation professionnelle, ce qui permettrait par la suite de mieux localiser les besoins des futurs criminologues.

\section{MÉTHODOLOGIE}

Pour retracer l'ensemble des bacheliers de 1970 à 1983, nous avons fait appel en premier lieu au Bureau du registraire de

* Le bachelier est un diplômé du premier cycle, dans les universités canadiennes.

** Michèle Dionne, auxiliaire de recherche, École de criminologie, Université de Montréal.

1. Tout au long de cette recherche, nous emploierons, pour des motifs de clarté et de concision, le genre masculin qui désigne aussi bien les hommes que les femmes. 
l'Université qui nous a fourni la liste des 1001 diplômés à rejoindre. Ensuite, nous avons consulté l'Association professionnelle des criminologues du Québec qui a mis à notre disposition une liste de 800 criminologues œuvrant dans différents secteurs professionnels. Nous avons pu retracer dans cette liste les adresses de 631 bacheliers. Manquaient encore les adresses des 370 autres diplômés n'y figurant pas. À cet effet, nous avons examiné les dossiers du secrétariat de l'École de criminologie et avons ainsi obtenu l'adresse probable de ces 370 diplômés.

La cueillette des données pour cette recherche s'est effectuée au moyen d'un questionnaire écrit, l'instrument de mesure se devant d'être concis puisque nous voulions rejoindre l'ensemble des diplômés et non seulement un échantillon.

Le questionnaire comprend une majorité de questions fermées avec toutefois dans plusieurs cas, une catégorie de réponse «autre».

Un premier envoi de questionnaires a eu lieu vers la fin d'avril 1984 et un mois plus tard, nous avons expédié une lettre de rappel afin d'obtenir le maximum de répondants.

Les sujets qui ont répondu au questionnaire sont au nombre de 546 , soit $55 \%$ de la population totale. Par ailleurs, 90 personnes n'ont pu être rejointes ( $9 \%$ de la population). Pour ces personnes, malgré plusieurs démarches, le questionnaire et/ou la lettre de rappel nous sont revenus avec la mention «adresse inexacte» ou «inconnu à cette adresse». Les 363 autres individus ( $36 \%$ de la population) ont probablement reçu le questionnaire, mais ont négligé d'y répondre.

Les classes de finissants qui accusent le plus faible taux de réponses sont celles des années 1970, 1972 et 1973.

\section{L'ÉCHANTILLON}

\section{LE SEXE}

Dans l'échantillon de 546 sujets, on retrouve 260 hommes et 286 femmes, soit des pourcentages respectifs de $48 \%$ et $52 \%$. Ces pourcentages présentent une équivalence parfaite avec la répartition par sexe de la population totale.

\section{$L^{\prime} \hat{A} G E$}

Les premiers bacheliers en criminologie n'ayant terminé leurs études qu'en 1970, on comprend que l'échantillon regroupe des 
gens relativement jeunes. Ainsi, $48 \%$ des sujets se situent entre 25 et 30 ans.

\section{LA FORMATION ACADÉMIQUE DES SUIETS}

Chacun des individus interrogés devait posséder au moins un baccalauréat en criminologie. Cependant, plusieurs d'entre eux ont acquis par la suite une formation criminologique supérieure, soit au niveau de la maîtrise, soit au niveau du doctorat.

Notre échantillon présente un pourcentage plus élevé de femmes détentrices d'un baccalauréat. Par contre, la proportion d'hommes à posséder une scolarité de maîtrise et une maîtrise est plus élevée. Cependant, alors que la proportion des hommes qui terminent une scolarité de maîtrise diminue d'année en année, cette proportion est plus instable chez les femmes avec une tendance à la hausse, depuis 1980. Par ailleurs, on remarque qu'il y a nettement plus d'hommes parmi les sujets ayant complété une maîtrise. Il ne faut cependant pas oublier que les gens qui sont ici rapportés comme ayant une formation de niveau supérieur en criminologie sont uniquement ceux qui possèdent déjà un baccalauréat en criminologie. C'est-à-dire que les sujets détenteurs d'une maîtrise en criminologie, d'un baccalauréat dans une autre discipline, ne sont pas recensés dans cette étude.

\section{LA PRINCIPALE ACTIVITÉ DES SUJETS}

Avant d'examiner le profil d'emplois des criminologues, il semblait essentiel de déterminer comment les criminologues définissent leur principale occupation actuelle. Considèrent-ils leur travail comme directement relié à la criminologie ou non? Sont-ils en chômage ou sans travail par choix? Sont-ils aux études?

Le bilan de l'activité principale actuelle des criminologues de notre échantillon montre que deux (2) individus sur trois (3) travaillent en criminologie, un (1) sur cinq (5) se retrouve dans un travail dans un autre domaine, près de un (1) sur dix (10) est en chômage ou sans travail par choix et finalement, un (1) sur vingt (20) poursuit des études en criminologie ou dans un autre domaine.

\section{LES CRIMINOLOGUES SUR LE MARCHÉ DU TRAVAIL}

a) Le temps écoulé avant d'arriver sur le marché du travail

D'une façon générale, les femmes prennent plus de temps à se trouver un emploi que les hommes et ce, quelle que soit l'année 
de graduation. Ainsi, pour les promotions 1970-1971 et 1978-1979, (selon le tableau 1) une forte proportion d'hommes ont trouvé un emploi en criminologie dès la fin de leurs études, alors que la proportion de femmes dans la même situation était de beaucoup inférieure. Par contre, chez les gens qui ont eu à attendre un à six mois avant de se trouver un emploi en criminologie, on remarque que ce sont les femmes qui sont proportionnellement plus représentées.

En outre, on retrouve une forte proportion des sujets qui ont terminé leurs études depuis 1980, qui ont mis sept (7) mois et plus pour se trouver un emploi.

Il semble donc qu'en général, les gens mettent de plus en plus de temps à trouver du travail et cette situation est particulièrement marquée pour les femmes qui ont toujours trouvé des emplois plus tardivement que les hommes.

TABLEAU 1

Temps écoulé avant le premier emploi en criminologie selon le sexe et l'année de graduation

\begin{tabular}{|c|c|c|c|c|c|c|c|c|c|c|c|c|c|c|c|}
\hline \multirow{3}{*}{$\begin{array}{c}\text { Année } \\
\text { de } \\
\text { graduation }\end{array}$} & \\
\hline & \multicolumn{4}{|c|}{ Aucun } & \multicolumn{4}{|c|}{$\begin{array}{l}\text { NOMBRE DE } \\
1 \text { à } 6\end{array}$} & \multicolumn{4}{|c|}{7 et plus } & \multicolumn{3}{|c|}{ Total } \\
\hline & $\stackrel{\text { Hom }}{N}$ & nmes & & $\underset{\%}{\text { mmes }}$ & $\stackrel{\text { Hom }}{\mathrm{N}}$ & nmes & & $\begin{array}{c}\text { nmes } \\
\%\end{array}$ & $\stackrel{\text { Hon }}{\mathrm{N}}$ & $\begin{array}{l}\text { mmes } \\
\%\end{array}$ & & $\begin{array}{c}\text { nmes } \\
\%\end{array}$ & $\begin{array}{l}\text { Hommes } \\
N\end{array}$ & & $\begin{array}{c}\text { nmes } \\
\%\end{array}$ \\
\hline $1970-1971$ & 20 & 65 & 5 & 42 & 11 & 35 & 6 & 50 & 0 & $\mathbf{0}$ & 1 & 8 & 31100 & 12 & 100 \\
\hline 1972.1973 & 7 & 50 & 8 & 44 & 7 & 50 & 9 & 50 & 0 & 0 & 1 & 6 & 14100 & 18 & 100 \\
\hline 1974.1975 & 23 & 62 & 15 & 56 & 13 & 35 & 10 & 37 & 1 & 3 & 2 & 7 & 37100 & 27 & 100 \\
\hline $1976-1977$ & 20 & 46 & 13 & 35 & 23 & 52 & 19 & 51 & $\mathbf{1}$ & 2 & 5 & 14 & 44100 & 37 & 100 \\
\hline $1978-1979$ & 26 & 47 & 13 & 37 & 12 & 31 & 12 & 34 & 1 & 2 & 10 & 29 & 39100 & 35 & 100 \\
\hline $1980-1981$ & 13 & 41 & 16 & 26 & 15 & 47 & 32 & 52 & 4 & 12 & 14 & 22 & 32100 & 62 & 100 \\
\hline 1982.1983 & 17 & 46 & 17 & 33 & 11 & 30 & 18 & 34 & 9 & 24 & 17 & 33 & 37100 & 52 & 100 \\
\hline Total & 125 & 54 & 87 & 36 & 92 & 39 & 106 & 44 & 16 & 7 & 50 & 20 & 234100 & 243 & 100 \\
\hline
\end{tabular}

\section{b) Le premier emploi à la fin des études}

Les criminologues ont occupé, au cours de leur carrière, plusieurs emplois différents. C'est pourquoi nous avons effectué des regroupements de types d'emplois.

Sept grandes catégories d'emplois ont été créées: 1) La catégorie «fédéral» qui désigne tout emploi qui s'exerce dans un pénitencier, aux libérations conditionnelles, au ministère du Sollioiteur général ou tout emploi relevant du gouvernement fédéral et relié à la criminologie. 2) La catégorie "provincial ministère de la Justice» réfère aux emplois au niveau de la proba- 
tion, de la détention, de la Commission québécoise des libérations conditionnelles, de la C.E.C.O. ${ }^{1}$, de la S.Q. ${ }^{2}$ ou de projets de ce ministère. 3) Le groupe «provincial — ministère des affaires sociales» désigne les emplois qui s'accomplissent dans un C.S.S. ${ }^{3}$, un C.L.S.C. ${ }^{4}$, dans un centre d'accueil, à l'Institut Philippe Pinel, ou dans tout autre service relevant de ce ministère. 4) La catégorie «enseignement-recherche» réunit les gens dont les employeurs sont un C.E.G.E.P. ${ }^{5}$, une université, un centre de recherche affilié à une université ou un centre de documentation. 5) La catégorie «autres emplois en criminologie» indique des emplois variés qui relèvent de domaines spécialisés comme la toxicomanie, la victimologie, la prévention. Ces divers emplois ont cependant comme point commun de relever directement de la criminologie. 6) Le groupe «domaines connexes» désigne des emplois dans le domaine de la sécurité, de la surveillance, du travail social, du droit ou de la santé et psychiatrie. Enfin, 7) la catégorie "autres domaines» représente tous les emplois qui n'ont vraiment aucun lien avec la criminologie comme par exemple, les emplois dans le domaine du commerce, dans le domaine scolaire, dans le domaine artistique, etc.

Le tableau 2 permet de constater que les criminologues se trouvent majoritairement un premier emploi aux gouvernements fédéral ou provincial (ministère de la Justice et M.A.S.6). Mais cette tendance s'atténue avec les années et depuis 1980-1981, plus de sujets obtiennent leur premier emploi dans les catégories «domaines connexes» et «autres domaines». On constate une stabilité, peu importe l'année, au niveau du premier emploi dans la catégorie "fédéral». En effet, entre $20 \%$ et $30 \%$ de diplômés ont eu comme premier employeur le gouvernement du Canada, de 1970 à 1983.

La nature du premier emploi varie selon le sexe des diplômés. Ainsi, les femmes qui se trouvent un premier emploi au M.A.S.

1. Commission d'enquête sur le crime organisé.

2. Sûreté du Québec.

3. Centre services sociaux.

4. Centre local de services communautaires.

5. Collège d'enseignement général et professionnel.

6. Ministère des Affaires sociales (Québec). 
TABLEAU 2

Nature du premier emploi selon le sexe et l'année de graduation

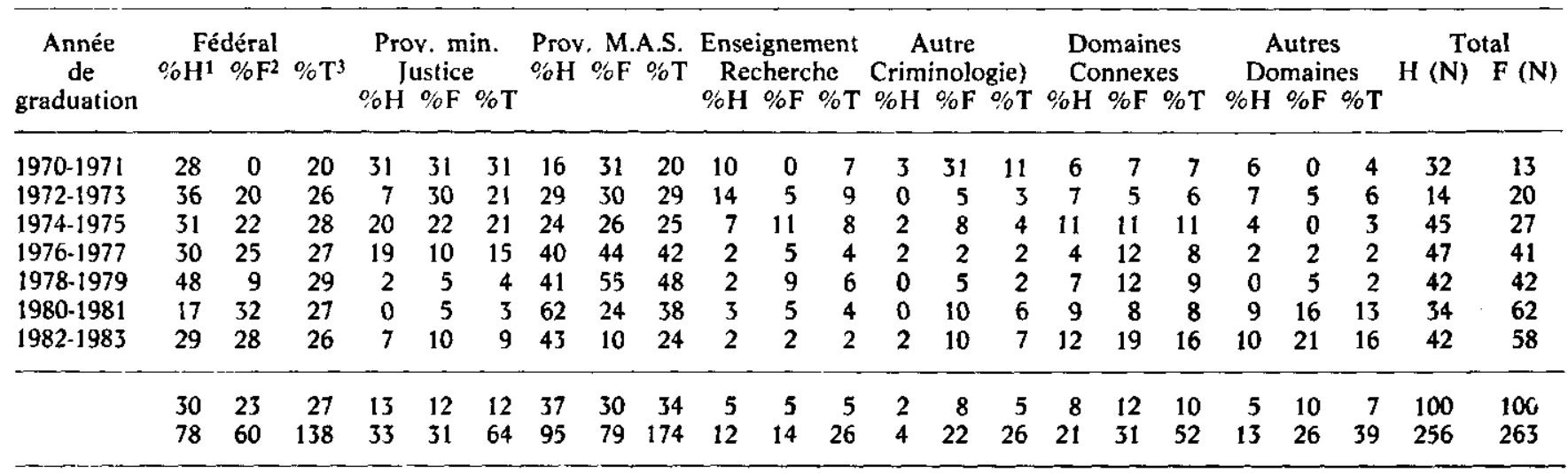

$1-\%$ des hommes

2 - \% des femmes

$3-\%$ total pour la catégorie 
sont de moins en moins nombreuses depuis 1980-1981, alors qu'elles y avaient toujours été les plus nombreuses auparavant. En plus, elles ont un avantage numérique sur les hommes dans les catégories «autres emplois en criminologie», «domaines connexes» ou «autres domaines» et ce, surtout depuis 1976-1977. En 1970 et 1971, aucune femme n'a obtenu un premier emploi au «fédéral», alors que $28 \%$ des hommes en avaient un. En 1972, une femme a obtenu un premier emploi au «fédéral», c'est donc lentement que ce dernier a accepté les femmes sans expérience.

\section{c) Le deuxième emploi}

Des 546 sujets de l'échantillon, 282 d'entre eux, (52\%) ont quitté leur premier emploi pour un autre travail. Si un peu plus de la moitié des criminologues changent d'emploi après un certain temps, cela implique aussi que presque la moitié des diplômés restent stables à cet égard. Cependant, on est en droit de supposer que la stabilité réelle des diplômés est moins grande que notre échantillon ne le montre puisque chez ceux qui n'ont pas répondu au questionnaire, se trouve probablement un grand nombre de diplômés qui ont quitté la criminologie après une première expérience de travail dans ce domaine.

Ce qui demeure évident pour le deuxième emploi, c'est une concentration des sujets dans les trois mêmes catégories principales, soit le gouvernement fédéral, le gouvernement provincial ministère de la Justice et le M.A.S. Dans l'ensemble, on constate peu de différence selon les années, dans la répartition des sujets, entre le premier emploi et le deuxième. Si une certaine proportion d'individus s'est déplacée vers le "fédéral» en 1980-1981, beaucoup sont allés travailler dans d'autres domaines pour les mêmes années. Tandis qu'en 1982-1983, alors que la catégorie «fédéral» accueillait, pour le deuxième emploi, une très bonne proportion des sujets, les catégories «autres domaines» et «domaines connexes» voyaient décroître leur pourcentage de sujets.

Les années terminales de 1970-1971, 1972-1973 montrent également des particularités intéressantes. Premièrement, les répondants de ces années ne représentent que $15 \%$ de l'ensemble des bacheliers. On remarque de plus que sur 282 personnes qui ont dit avoir eu un deuxième emploi, on en retrouve $20 \%$ qui ont terminé en 1970-1971, 1972-1973. C'est donc dire que les sujets qui ont obtenu leur diplôme au cours de ces années ont majoritai- 
rement connu un deuxième emploi. En plus, c'est vers un domaine connexe ou vers un autre domaine que les gens semblent s'être déplacés puisque ces catégories voient leurs pourcentages de sujets augmenter considérablement.

Le sexe des sujets est déterminant dans le deuxième domaine d'emploi. Par exemple en 1976-1977 et 1978-1979, alors qu'il y a autant d'hommes que de femmes au M.A.S., le reste des sujets se répartit fort différemment dans les autres catégories. La majorité des femmes s'orientent dans les domaines «enseignementrecherche», «autre emploi en criminologie», "domaines connexes» et "autres domaines». Mais chez les hommes, ces catégories recueillent peu de sujets et après le M.A.S., c'est au «fédéral» ou au "provincial - ministère de la Justice» qu'on retrouve les hommes dans leur deuxième emploi.

Au premier emploi, il n'y avait que $10 \%$ de l'ensemble des femmes qui travaillaient dans un "autre domaine» alors qu'au deuxième, ce pourcentage est de $17 \%$. Cette situation ne peut que paraître inquiétante, mais persistera-t-elle ou sera-t-elle en régression? Une seule variable permettra d'évaluer grossièrement cette situation et c'est celle qui décrit le type de l'emploi actuel, si le sujet a eu plus de deux emplois. Voyons donc la situation quant à l'emploi actuel des gens qui ont eu plus de deux emplois.

\section{d) L'emploi actuel si le sujet a eu plus de deux emplois}

Parmi les 546 sujets de l'échantillon, il n'y en a que 140 , soit $23 \%$ qui ont eu plus de deux emplois et qui ont consenti à décrire le type d'emploi qu'ils avaient actuellement. Parmi ceux-ci, on compte $49 \%$ d'hommes. Le petit nombre de sujets présents dans chacune des catégories d'emplois ne permet qu'une étude sommaire de la situation.

La comparaison entre l'emploi actuel et le deuxième emploi (si le sujet a eu plus de deux emplois) montre que les emplois actuels sont en hausse dans la catégorie «domaines connexes» surtout pour les femmes, mais également aussi pour les hommes. On retrouve moins de gens qui y ont un emploi au M.A.S., et ce, autant chez les hommes que chez les femmes. Par contre, on constate une forte augmentation du pourcentage des hommes dans la catégorie "autres domaines》 et particulièrement chez ceux qui ont terminé leur baccalauréat de 1970 à 1971. Il y a proportion- 
nellement moins d'hommes et de femmes qui occupent actuellement un emploi au fédéral qu'il y en avait lors du deuxième emploi.

\section{LES FONCTIONS DES CRIMINOLOGUES AU TRAVAIL}

Avant de procéder à la description des fonctions des criminologues, définissons les catégories de fonctions que nous avons formées. La catégorie "fonction clinique» est indépendante et nécessite peu de précision quant à sa définition. Par contre, dans la catégorie «autres fonctions en criminologie», toutes les fonctions reliées à l'administration, la recherche, l'enseignement, la documentation ou l'analyse de documents ont été regroupées. Elles se situent cependant toujours dans le contexte de la criminologie. Les "autres fonctions dans un domaine connexe» sont celles qui s'exercent dans des domaines aussi variés que la sécurité (agent de correction, policier), la sociologie (enseignement) et la bibliothéconomie. Ces fonctions font ou peuvent faire appel, à des degrés divers, à la criminologie. Quant au dernier groupe de fonctions, il n'exige aucune notion de criminologie. Le groupe «autres fonctions, autre domaine» rassemble des fonctions qui relèvent des domaines de l'administration (conseiller fiscal, comptable), du commerce (caissière, vendeur, etc.), de la construction (électricien, menuisier) ou tout autre domaine allant du théâtre, à l'agriculture, et à l'ébénisterie.

D'après le tableau 3, lors du premier emploi, il y a presque autant de femmes que d'hommes dans les fonctions directement reliées à la criminologie, mais on retrouve deux fois plus de femmes que d'hommes occupant une fonction non clinique.

Les gens qui ont eu plus d'un emploi abandonnent peu à peu les fonctions de nature clinique et ceci particulièrement chez les hommes. Les catégories "autres fonctions en criminologie» et "autres fonctions dans un autre domaine» vont chercher des pourcentages plus élevés de sujets masculins $(41 \%$ et $16 \%$ respectivement) lorsque ceux-ci ont eu plus de deux emplois. Toutefois, chez les femmes, la fonction clinique perd moins de sa popularité et même si les catégories "autres fonctions en criminologie» et «autres fonctions dans un domaine connexe» connaissent une augmentation (30\% et $18 \%$ respectivement), cette dernière est moins marquée que chez les hommes. 


\section{TABLEAU 3}

Répartition (\%) des sujets d'après leurs fonctions lors de leur premier emploi, deuxième emploi ou de leur emploi actuel selon le sexe

\begin{tabular}{|c|c|c|c|c|c|c|c|c|c|c|c|c|}
\hline \multirow[b]{2}{*}{ Sexe } & \multicolumn{3}{|c|}{$\begin{array}{l}\text { Fonction } \\
\text { clinique } \\
\text { en } \\
\text { criminologie } \\
\text { Emploi }\end{array}$} & \multicolumn{3}{|c|}{$\begin{array}{c}\text { Autres } \\
\text { fonctions } \\
\text { en } \\
\text { criminologie } \\
\text { Emploi }\end{array}$} & \multicolumn{3}{|c|}{$\begin{array}{l}\text { Autres } \\
\text { fonctions } \\
\text { domaine } \\
\text { connexe } \\
\text { Emploi }\end{array}$} & \multicolumn{3}{|c|}{$\begin{array}{l}\text { Autres } \\
\text { fonctions } \\
\text { autres } \\
\text { domaine } \\
\text { Emploi }\end{array}$} \\
\hline & fer & $2 \mathrm{e}$ & actuel & fer & $2 \mathrm{e}$ & actuel & ger & $2^{e}$ & actue & Ier & $2^{e}$ & actue \\
\hline Masculin & 77 & 60 & 33 & 12 & 22 & 41 & 7 & 9 & 10 & 4 & 9 & 16 \\
\hline Féminin & 59 & 54 & 48 & 25 & 22 & 30 & 6 & 9 & 18 & 10 & 15 & 4 \\
\hline
\end{tabular}

D'autre part (chez les diplômés qui ont eu plus de deux emplois), les fonctions occupées dans l'emploi actuel sont, pour plusieurs, des fonctions non cliniques, mais dans le domaine de la criminologie. Ainsi, $41 \%$ des hommes qui ont eu plus de deux emplois, occupent ce type de fonction contre $30 \%$ des femmes.

En conclusion, retenons que le sexe des sujets est déterminant dans la nature des fonctions des individus. L'année de la fin des études est également un élément influant sur le type de fonction occupée, mais à un degré moindre. Cela est particulièrement évident pour le premier emploi obtenu en fin d'études. En effet, depuis 1980, les nouveaux diplômés sont moins nombreux à occuper une fonction clinique en criminologie. Ils occupent plutôt d'autres fonctions en criminologie ou dans un domaine connexe.

\section{LE PROFIL OU L'ÉVOLUTION DES EMPLOIS CHEZ LES CRIMINOLOGUES}

La description des emplois occupés est déjà un bon indicateur de la situation des criminologues sur le marché du travail. Mais voyons aussi comment les sujets planifient leur carrière, c'est-à-dire quel emploi délaissent-ils au profit de quel autre?

D'abord, nous tenterons de déterminer dans quel domaine travaillent les gens qui ont quitté leur premier emploi et quelle est la proportion des hommes et des femmes qui effectuent de tels changements. Ensuite, nous décrirons dans quelle direction s'effectue le changement du premier au deuxième emploi.

a) Les sujets qui quittent leur premier emploi

Comme l'indique le tableau 4 , les femmes quittent plus fréquemment leur premier emploi. Règle générale, les différences 
de mobilité selon le sexe ne sont pas significatives selon le domaine du travail. Seules les femmes travaillant dans «autres emplois en criminologie» et «domaines connexes» ont quitté leur prenier emploi beaucoup plus souvent que les hommes. Il semble aussi que celles qui travaillaient au ministère des Affaires sociales sont un peu plus mobiles que les hommes à l'emploi de ce même ministère, puisque $62 \%$ d'entre elles ont quitté leur premier emploi comparativement à $51 \%$ des hommes.

\section{b) Du premier emploi au deuxième emploi}

Puisque plus de la moitié des sujets ont changé d'emploi, il est essentiel de voir dans quelle autre catégorie d'emploi ces gens se dirigent.

Pour les trois catégories qui regroupent le plus de sujets, soit le «fédéral», le "provincial - ministère de la Justice» et le "provincial - ministère des Affaires sociales», les constatations suivantes s'imposent. Comme on peut le voir à la figure 1, plusieurs sujets ont quitté un emploi au «fédéral» pour occuper un emploi différent, mais encore au «fédéral». Le même phénomène se produit pour le provincial - M.A.S. C'est donc dire que si les gens changent de type d'emploi, ils conservent souvent le même employeur.

Par contre, les gens qui ont quitté un emploi au «provincial ministère de la Justice», ont acquis des emplois tant au "fédéral», au «provincial - M.A.S.» qu'au "provincial - ministère de la Justice». Ces constatations pour les trois principales catégories d'emploi sont valables autant pour les hommes que pour les femmes.

Les catégories "enseignement-recherche», «autre emploi en criminologie», "domaine connexe» et «autre domaine» montrent des mouvements beaucoup plus variés. Ainsi, les sujets qui ont travaillé dans le domaine «enseignement-recherche» lors d'un premier emploi, semblent se trouver un deuxième emploi principalement au "provincial - M.A.S.», dans un «domaine connexe» ou au «fédéral». Il faut noter ici que le peu de sujets (13 seulement) ayant un premier emploi dans cette catégorie «enseignementrecherche» et un deuxième emploi dans une autre catégorie, nous oblige à interpréter prudemment ces mouvements.

Les sujets qui avaient un premier emploi dans la catégorie «autre emploi en criminologie» s'orientent principalement vers le 


\section{FIGURE 1}

Pourcentage des gens qui passent d'un premier emploi à un deuxième emploi différent pour les cinq principales catégories d'entoloi

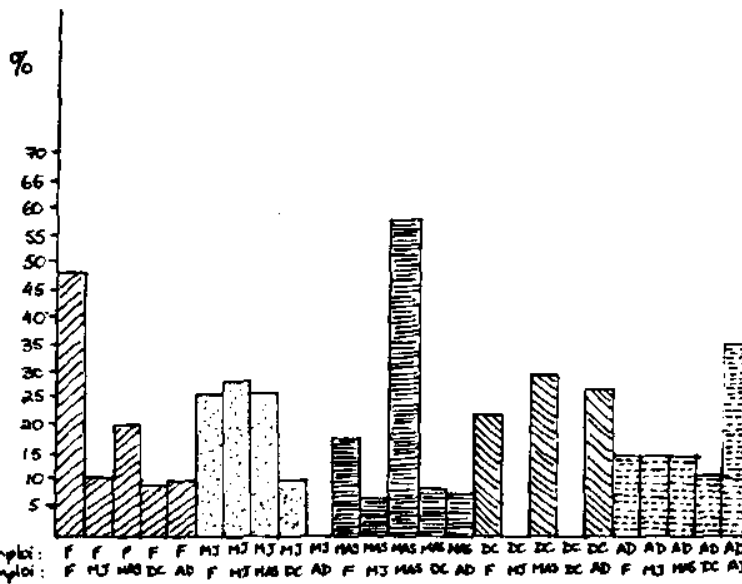

Légende :

F : fédéral

M.J. : provincial, ministère de la Justice

M.A.S. : provincial, ministère des Affaires sociales

D.C. : domaine connexe

A.D. : autre domaine

\section{TABLEAU 4}

Sujets qui ont quitté leur premier emploi d'après le sexe et le domaine d'emploi

\begin{tabular}{lrrrrrr}
\hline & \multicolumn{2}{c}{ Hommes } & \multicolumn{2}{c}{ Femmes } & \multicolumn{2}{c}{ Total } \\
Domaine & N & $\%$ & N & $\%$ & H & F \\
\hline Fédéral & 48 & 62 & 36 & 60 & 78 & 60 \\
Prov. ministère de la Justice & 16 & 48 & 17 & 55 & 33 & 31 \\
Prov. M.A.S. & 48 & 51 & 49 & 62 & 95 & 79 \\
Enseignement-Recherche & 8 & 67 & 9 & 64 & 12 & 14 \\
Autres (criminologie) & 2 & 50 & 18 & 82 & 4 & 22 \\
Domaines connexes & 6 & 29 & 18 & 58 & 21 & 31 \\
Autres domaines & 10 & 77 & 18 & 69 & 13 & 26 \\
\hline
\end{tabular}

gouvernement fédéral ou dans «l'enseignement-recherche» au deuxième emploi. Quelques-uns se retrouvent également au «provincial - M.A.S.» ou dans un «autre domaine». 
Aucun des sujets qui avaient un premier emploi dans un "domaine connexe» n'a obtenu un deuxième emploi dans cette catégorie. Ces sujets auraient donc abouti dans cette catégorie d'emploi sans avoir eu le choix. Mais dès qu'ils ont pu changer d'emploi et aller vers la criminologie, ils ont profité de cette chance. Ainsi, au deuxième emploi, ces sujets se répartissent presque également dans les catégories «fédéral», "provincial M.A.S.», «autre emploi en criminologie» et «autre domaine».

Finalement, le tiers des individus qui avaient un premier emploi dans la catégorie "autre domaine» ont eu un deuxième emploi dans la même catégorie alors que les autres sujets se trouvaient un deuxième emploi, soit au «fédéral», au "provincial - ministère de la Justice» ou «M.A.S.» et dans un «autre domaine».

\section{MOUVEMENT DANS LES EMPLOIS}

Un changement d'emploi peut indiquer soit une insatisfaction face au travail, des attentes non comblées, ou encore une carrière en progression. Il ne s'agit pas ici d'interpréter les motifs des changements d'emploi des criminologues, mais de décrire leur comportement au niveau de la mobilité dans l'emploi. Pour ce faire, nous déterminerons simplement le nombre d'emplois que les criminologues ont occupés depuis qu'ils ont quitté leur premier emploi.

D'après le tableau 5 , il y a 310 sujets $(57 \%)$ qui ont eu plus d'un emploi et qui se sont prononcés sur le nombre d'emplois qu'ils avaient eus depuis le premier.

On doit être prudent dans l'interprétation de ces données, car elles peuvent signifier des réalités différentes selon l'année d'obtention du baccalauréat. Ainsi, chez les individus qui ont terminé depuis 15 ans, le fait d'occuper plusieurs emplois successivement peut indiquer une carrière professionnelle qui progresse sans cesse. Par contre, ceux qui ont changé deux ou trois fois d'emplois depuis 1980 , année de leur baccalauréat, n'ont peut-être pas réussi à trouver un emploi dans leur domaine. ou ils n'ont occupé jusqu'à maintenant que des emplois temporaires comme des contrats de quelques mois. On doit se rappeler que le marché du travail a considérablement évolué de 1970 à 1983 et que les criminologues n'ont pas échappé à cette évolution. 


\begin{tabular}{|c|c|c|c|c|c|c|c|c|c|c|c|c|c|c|c|c|c|c|}
\hline \multirow{2}{*}{$\begin{array}{c}\text { Annte } \\
\text { de } \\
\text { graduation }\end{array}$} & \multicolumn{3}{|c|}{ Aucun } & \multicolumn{3}{|c|}{1} & \multicolumn{3}{|c|}{2} & \multicolumn{3}{|c|}{3} & \multicolumn{3}{|c|}{4 et plus } & \multicolumn{3}{|c|}{ Total } \\
\hline & $\begin{array}{c}H^{1} \\
N(\%)\end{array}$ & $\begin{array}{c}F^{2} \\
N(\%)\end{array}$ & $\begin{array}{c}T^{3} \\
N(\%)\end{array}$ & $\begin{array}{c}\mathbf{H}^{1} \\
\mathbf{N}(\%)\end{array}$ & $\begin{array}{c}F^{2} \\
N(\%)\end{array}$ & $\begin{array}{c}\mathrm{T}^{\mathbf{3}} \\
\mathrm{N}(\%)\end{array}$ & $\begin{array}{c}\mathbf{H}^{\mathbf{1}} \\
\mathrm{N}(\%)\end{array}$ & $\begin{array}{c}F^{2} \\
N(\%)\end{array}$ & $\begin{array}{c}T^{3} \\
N(\%)\end{array}$ & $\begin{array}{c}\mathbf{H}^{1} \\
N(\%)\end{array}$ & $\begin{array}{c}F^{2} \\
\left.N^{2} \%\right)\end{array}$ & $\stackrel{\mathbf{T}^{\mathbf{3}}}{\mathbf{N}(\%)}$ & $\begin{array}{c}\mathbf{H}^{\mathbf{1}} \\
\mathrm{N}(\%)\end{array}$ & $\begin{array}{c}F^{2} \\
N(\%)\end{array}$ & $\begin{array}{c}T^{\mathbf{3}} \\
N(\%)\end{array}$ & $\begin{array}{c}\mathbf{H}^{\mathbf{1}} \\
\mathbf{N}(\%)\end{array}$ & $\begin{array}{c}F^{2} \\
N(\%)\end{array}$ & $\begin{array}{c}T^{3} \\
N(\%)\end{array}$ \\
\hline 1970.1971 & $O(0)$ & $0(0)$ & $O(0)$ & $6(30)$ & $5(46)$ & $11(36)$ & $2(10)$ & $2(18)$ & $4(13)$ & $4(20)$ & $3(27)$ & $7(22)$ & $8(40)$ & $1(9)$ & $9(29)$ & $137(44)$ & $173(56)$ & $310(00)$ \\
\hline $1972-1973$ & $0(0)$ & $2(12)$ & $2(8)$ & $1(11)$ & $3(19)$ & $4(16)$ & $3(33)$ & $5(31)$ & $B(32)$ & $2(22)$ & $4(25)$ & $6(24)$ & $3(33)$ & $2(13)$ & $5(20)$ & 20 & 11 & 31 \\
\hline $1974-1975$ & $1(8)$ & $1(6)$ & $2(5)$ & $9(35)$ & $8(47)$ & $17(39)$ & $7(27)$ & $4(23)$ & $11(27)$ & $4(15)$ & $3(18)$ & $7(16)$ & $5(19)$ & $1(16)$ & $6(14)$ & 9 & 16 & 25 \\
\hline $1976-1977$ & $o(0)$ & $2(7)$ & $2(4)$ & $12(52)$ & $12(41)$ & $24(46)$ & $6(26)$ & $11(38)$ & $17(33)$ & $3(13)$ & $4(14)$ & $7(13)$ & $2(9)$ & $O(0)$ & $2(4)$ & 26 & 17 & 43 \\
\hline [978-1979 & $0(0)$ & $1(15)$ & $1(2)$ & $11(52)$ & $6(32)$ & $17(43)$ & $4(19)$ & $8(42)$ & $12(30)$ & $5(24)$ & $D(0)$ & $5(13)$ & $1(5)$ & $4(21)$ & $5(12)$ & 23 & 29 & 52 \\
\hline $1980-1981$ & $1(5)$ & $t(2)$ & $2(3)$ & $9(43)$ & $18(43)$ & $27(43)$ & $5(24)$ & $11(26)$ & $16(25)$ & $3(14)$ & $8(19)$ & $11(18)$ & $3(14)$ & $4(10)$ & $7(t 1)$ & 21 & 19 & 40 \\
\hline $1982-1983$ & $1(6)$ & $7(18)$ & $8(14)$ & $14(42)$ & $19(49)$ & $33(59)$ & $1(6)$ & $9(23)$ & $10(18)$ & $O(0)$ & $2(5)$ & $2(4)$ & $1(6)$ & $2(5)$ & $3(5)$ & 21 & 42 & 63 \\
\hline & $3(2)$ & $14(8)$ & $17(5)$ & $62(45)$ & $71(41)$ & $133(43)$ & $28(21)$ & $50(29)$ & $78(25)$ & $78(25)$ & $21(15)$ & $45(15)$ & $33(17)$ & $14(8)$ & $37(2)$ & 17 & 39 & 56 \\
\hline
\end{tabular}

1 - Hommes

2 - Femmes

3 - Total

TABLEAU 6

Les périodes de temps sans travail en criminologie selon le sexe des suiets

\begin{tabular}{|c|c|c|c|c|c|c|c|c|c|c|c|c|c|c|}
\hline $\begin{array}{l}\text { Genre } \\
\text { d'inactivite } \\
\text { en criminologie }\end{array}$ & $\mathrm{H}^{\mathrm{H}(\%)}$ & $\begin{array}{l}\text { aais } \\
\mathbf{N}(\%)\end{array}$ & $\begin{array}{c}t \% \\
H \\
N(\%)\end{array}$ & $\begin{array}{c}25 \% \\
\mathrm{~F} \\
\mathrm{~N}(\%) \\
\end{array}$ & $\begin{array}{c}26 \% \\
\text { H } \\
\text { N(\%) }\end{array}$ & $\begin{array}{c}50 \% \\
F \\
N(\%)\end{array}$ & $\begin{array}{r}51 \% \\
H \\
N(\%) \\
\end{array}$ & $\begin{array}{c}75 \% \\
F \\
N(\%)\end{array}$ & $\begin{array}{c}76 \% \\
H \\
N(\%) \\
\end{array}$ & $\begin{array}{c}99 \% \\
\mathbf{F} \\
\mathbf{N}(\%)\end{array}$ & $\begin{array}{c}\mathrm{N}_{\mathrm{H}}(\%) \\
.\end{array}$ & $\mathrm{N}(\%)$ & $\underset{N(\%)}{H^{T}}$ & $\underset{N(\%)}{F}$ \\
\hline \multirow{2}{*}{$\begin{array}{l}\text { Sens travail } \\
\text { par choix } \\
\text { En chômage } \\
\text { Dans un travail } \\
\text { en dehors de la } \\
\text { criminologie }\end{array}$} & $\begin{array}{l}178(83) \\
118(72)\end{array}$ & $\begin{array}{r}125(55) \\
79(48)\end{array}$ & $\begin{array}{l}32(15) \\
41(25)\end{array}$ & $\begin{array}{l}59(31) \\
63(38)\end{array}$ & $\begin{array}{l}2(1) \\
3(2)\end{array}$ & $\begin{array}{l}20(9) \\
16(10)\end{array}$ & $\begin{array}{l}0(0) \\
2(1)\end{array}$ & $\begin{array}{l}7(3) \\
6(3)\end{array}$ & $\begin{array}{l}1(1) \\
0(0)\end{array}$ & $\begin{array}{l}3(1) \\
0(0)\end{array}$ & $\begin{array}{l}0(0) \\
0(0)\end{array}$ & $\begin{array}{l}1(1) \\
2(1)\end{array}$ & $\begin{array}{l}213 \\
164\end{array}$ & $\begin{array}{l}225 \\
166\end{array}$ \\
\hline & $58(59)$ & $78(44)$ & 20(12) & $36(20)$ & $13(8)$ & $22(12)$ & $8(5)$ & $17(10)$ & $11(6)$ & $10(6)$ & $17(10)$ & $15(8)$ & 167 & 178 \\
\hline
\end{tabular}


Globalement, le mouvement dans les emplois chez les hommes est différent de celui chez les femmes. Ainsi, de façon générale, il y a plus de femmes $(8 \%)$ que d'hommes $(2 \%)$ qui n'ont plus travaillé depuis qu'elles (ils) ont quitté leur premier emploi. Dans l'ensemble, chez les finissants de 1980 à 1983, les femmes changent plus souvent d'emplois que les hommes. Cependant, chez les sujets qui ont terminé leurs études entre 1970 et 1977, on compte plus d'hommes que de femmes dans la catégorie «4 emplois et plus». En 1970-1971 et 1972-1973, le pourcentage des hommes dans cette catégorie est même égal ou supérieur à celui des catégories 1, 2 et 3 emplois. Cela signifie que bon nombre de ceux qui ont obtenu leur baccalauréat de 1970 à 1973, ont connu une forte mobilité dans l'emploi.

Néanmoins, pour les deux sexes, indépendamment de l'année (hormis pour 1972-1973), c'est la catégorie «un emploi» qui rassemble le plus fort pourcentage de sujets. On peut donc conclure qu'après avoir quitté un premier emploi, une grande proportion de criminologues se trouveront un seul autre emploi.

\section{LES PÉRIODES DE TEMPS SANS TRAVAIL EN CRIMINOLOGIE}

Afin d'avoir un aperçu général de la situation des criminologues sur le marché du travail, les répondants devaient déterminer le pourcentage du temps écoulé depuis la fin de leurs études où ils avaient été : a) sans travail par choix, b) en chômage et c) dans un travail en dehors de la criminologie. Le tableau suivant montre la situation de travail des criminologues. (Tableau 6).

\section{a) Période de temps sans travail par choix}

Alors qu'à une des questions précédentes portant sur l'emploi actuel, aucun homme n'a répondu être sans travail par choix, quelques-uns admettent ici avoir connu une certaine période de temps, depuis la fin de leurs études, pendant laquelle ils n'avaient pas travaillé et cela, par choix. Cependant, pour presque tous les hommes qui ont connu ce genre d'inactivité, cela n'a représenté que 1 à $25 \%$ du temps écoulé depuis la fin de leurs études. De même, près de la moitié des femmes ont indiqué avoir été sans travail par choix pendant une certaine période de temps depuis la fin de leurs études. Pour la plupart, cette période de temps variait de 1 à $25 \%$ du temps écoulé depuis l'obtention de leur diplôme. 
Mais, pour quelques-unes, cette période a été de $26 \%$ à $75 \%$ du temps écoulé depuis la fin de leurs études, alors que seulement une a été sans travail par choix pendant plus de $75 \%$ du temps écoulé après l'obtention de son diplôme. Si ces pourcentages de temps sans travail par choix sont examinés d'après l'année de graduation des sujets, on constate que peu de sujets qui ont gradué en 1970-1971 ont connu un fort pourcentage de leur temps pendant lequel ils ont été sans travail par choix. Par contre, plus on se rapproche dans le temps, plus le pourcentage de sujets qui sont sans travail par choix grandit. Cette augmentation est aussi largement due aux femmes. Cela reste logique puisque de 1970 à 1983 , il y a eu de plus en plus de femmes qui ont terminé leurs études en criminologie.

\section{b) Période de temps en chômage}

On retrouve presque les mêmes différences au niveau des sexes et des années de graduation chez les répondants pour les périodes de temps en chômage et pour celles sans travail par choix. Ainsi, il y a plus de femmes que d'hommes qui ont connu des périodes de chômage, soit de 1 à $75 \%$ du temps écoulé depuis la fin de leurs études. Cependant, il est possible que les personnes que nous n'avons pas pu rejoindre soient en grande partie en chômage. Cela pourrait donc faire varier les pourcentages des gens qui ont connu des périodes de chômage depuis la fin de leurs études.

\section{c) Période de temps dans un travail en dehors de la criminologie}

La différence entre hommes et femmes s'atténue de façon notoire lorsque l'on considère les périodes de temps dans un travail en dehors de la criminologie. Les pourcentages des femmes qui ont connu une période de temps dans un travail en dehors de la criminologie sont semblables à ceux notés dans les catégories en chômage et sans travail par choix. Cependant, une plus grande proportion de femmes que d'hommes ont indiqué avoir été, pendant plus de $25 \%$ du temps écoulé depuis l'obtention de leur diplôme, dans un travail en dehors de la criminologie. Pour les hommes, on note que, si plus d'hommes se sont retrouvés dans un travail en dehors de la criminologie plutôt qu'en chômage ou sans travail par choix, ils l'ont été pendant des périodes de temps considérablement longues. 
Contrairement aux périodes de temps sans travail par choix ou en chômage, l'année a peu influencé le pourcentage du temps pendant lequel les sujets ont été dans un travail en dehors de la criminologie. Par exemple, on retrouve proportionnellement, autant de finissants des années 1970-1971 qui ont connu 51 à $75 \%$ de leur temps d'emploi dans un travail en dehors de la criminologie qu'on en retrouve chez ceux qui ont gradué en 1982-1983.

\section{CONCLUSION}

La relance des bacheliers en criminologie devait permettre essentiellement l'évaluation de la situation des criminologues sur le marché du travail. En définissant la principale activité des criminologues au moment où ils répondent au questionnaire, nous constatons que près des deux tiers des sujets occupent un emploi directement relié à leur formation. On remarque cependant que chez ces sujets, il y a beaucoup plus d'hommes que de femmes. Pourtant, pour l'ensemble des diplômés en criminologie (autant dans l'échantillon que dans la population), il y a légèrement plus de femmes que d'hommes.

Par ailleurs, les gens, après la fin de leurs études, accèdent de moins en moins rapidement au marché du travail. Il s'écoule de plus en plus de mois avant que les nouveaux diplômés se trouvent un emploi. Les femmes mettent généralement plus de temps à trouver leur premier emploi.

Le premier emploi est principalement offert par le gouvernement fédéral ou le gouvernement provincial - ministère des Affaires sociales et, un peu moins fréquemment, le ministère de la Justice du Québec. Cette situation est valable presque autant pour les femmes que pour les hommes. Par contre, les emplois dans des domaines connexes ou dans d'autres domaines que la criminologie sont occupés majoritairement par des femmes.

Lorsque les criminologues quittent leur premier emploi, c'est dans les mêmes ministères qu'ils évoluent principalement. Ils auront donc un deuxième emploi au «fédéral» ou au "provincial» pour le ministère des Affaires sociales ou de la Justice. Il est intéressant de constater qu'il y a peu de changement d'un ministère à l'autre.

Les fonctions des criminologues dans le cadre de ces emplois sont variées mais majoritairement de type clinique, surtout lors du 
premier emploi. Au deuxième emploi, les sujets délaissent un peu le côté clinique pour favoriser l'administration, la recherche ou l'enseignement, mais cela, toujours dans leur discipline.

La majorité des criminologues changent d'emploi, mais la mobilité est grande pour quelques-uns d'entre eux seulement. La plupart des sujets ont néanmoins occupé un seul autre emploi depuis le premier qu'ils ont eu. Cette constatation vaut pour la presque totalité des années de fin d'études et aussi quel que soit le sexe des sujets.

Nous pouvons donc tracer un portrait-robot du criminologue sur le marché du travail. Ainsi, il s'est trouvé un premier emploi soit au "fédéral» ou au "provincial» (M.A.S. ou ministère de la Justice). Il exerce une fonction clinique et lorsqu'il quitte cet emploi, il se trouve un autre emploi dans un des trois mêmes domaines mais il change de fonction. Cette fonction sera toutefois encore reliée à la criminologie.

En outre, il a été très rarement sans travail mais par contre il a connu des périodes de temps pendant lesquelles il a travaillé dans un autre domaine que la criminologie.

Enfin, retenons deux dimensions principales de la situation des criminologues sur le marché du travail. La première est que les criminologues n'échappent pas aux difficultés du marché du travail. Les fluctuations dans leurs emplois seront tributaires de la situation économique qui prévaut dans la société en général. Ainsi, les périodes de chômage seront plus nombreuses chez les gens qui ont terminé en 1982, qu'en 1970. Les diplômés de 1982-1983 mettront plus de temps à se trouver un emploi que ceux du début des années 1970 et ainsi de suite.

La deuxième dimension regarde les femmes. Quoiqu'elles soient un peu plus nombreuses que les hommes à avoir obtenu leur baccalauréat en criminologie, elles occupent beaucoup moins de place sur le marché du travail que les hommes. On les retrouvera donc plus nombreuses dans des domaines connexes ou en dehors de la criminologie. Cela signifie-t-il qu'elles se découragent plus vite devant les difficultés à accéder au poste qui convient à leur formation? Les politiques d'embauche sont-elles discriminatoires envers les femmes? Sont-elles si critiques ou polyvalentes que devant la non-satisfaction de leur idéal, elles n'hésitent pas à s'orienter vers d'autres domaines? Quoiqu'il en soit, les femmes 
évoluent différemment des hommes sur le marché du travail en criminologie.

Cette étude soulève au moins autant de questions qu'elle en résout. En permettant d'évaluer la situation des criminologues sur le marché du travail, nous débouchons, en prolongement, sur les problèmes de leur formation et de la définition de toute la profession. Mais ceci est une toute autre histoire.

\section{BIBLIOGRAPHIE}

BOIVIN, D., C. OUELLETTE (1977), l'École de criminologie: des étudiants s'interrogent, Université de Montréal, École de criminologie.

DE TROY, C. (1977), l'École de criminologie: les professeurs s'interrogent, Université de Montréal, École de criminologie.

GAGNON, R. (1977), l'École de criminologie: les étudiants et les praticiens, Université de Montréal, École de criminologie.

GAGNON, R. (1977), «Les praticiens de la criminologie au Québec», Criminologie, $\mathrm{X}, 2$ : 39-54. 